\title{
The Glycemic Index of Sport Nutrition Bars Affects Performance and Metabolism During Cycling and Next-Day Recovery
}

\author{
by \\ Mojtaba Kaviani ${ }^{1}$ Philip D. Chilibeck1, Jennifer Jochim¹, Julianne Gordon ${ }^{1}$, \\ Gordon A. Zello ${ }^{2}$
}

\begin{abstract}
Low-glycemic index carbohydrates are potentially better for endurance performance as they result in greater fat oxidation and lower carbohydrate oxidation due to lower insulin release. We compared the effects of pre-exercise feeding with a low-glycemic index lentil-based sports nutrition bar, a commercially-available sports nutrition bar with moderateglycemic index, and a non-caloric placebo on metabolism and performance during endurance cycling (Trial 1). Using a randomized, counterbalanced, crossover design, endurance-trained individuals ( $n=11$; eight males; $26 \pm 6 y$; VO2peak $51.4 \pm 1.6 \mathrm{~mL} / \mathrm{kg} / \mathrm{min}$ ) consumed $1.5 \mathrm{~g} / \mathrm{kg}$ available carbohydrate from a lentil bar and a moderate-glycemic index bar, as well as a placebo, $1 \mathrm{~h}$ before endurance cycling $(75 \mathrm{~min}$ at $65 \%$ VO2peak, followed by a $7 \mathrm{~km}$ time trial). We also compared post-exercise consumption of the low-glycemic index bar with another moderate-glycemic index bar on next-day exercise performance as an assessment of recovery (Trial 2). In Trial 1, fat or carbohydrate oxidation rates were not different between the bar conditions $(p>0.05)$. Blood lactate was lower during the low-versus the moderate-glycemic index condition after 75 minutes of cycling $(2.6$ versus $4.0 \mathrm{mmol} / \mathrm{L}, p<0.05)$ and at the end of the time trial (7.4 versus 9.1 $m m o l / L, p<0.05)$. Time trial performance improved $(p<0.05)$ after consumption of the low- $(574 \pm 55 s)$ and moderateglycemic index (583 \pm 59 s) bars compared to the placebo (619 \pm 81 s). In Trial 2 (next-day recovery), performance improved ( $p<0.05$ ) with the low-glycemic index bar (547 \pm 42 s) compared to the moderate-glycemic index bar (569 \pm $42 \mathrm{~s})$ and the placebo (566 $\pm 34 \mathrm{~s})$. Low- and moderate-glycemic index sports nutrition bars improved cycling exercise performance; however, only the low-glycemic index bar improved next day performance.
\end{abstract}

Key words: carbohydrate, exercise, fatigue, endurance.

\section{Introduction}

Carbohydrate consumption is important for endurance performance and recovery from endurance exercise (Litte et al., 2009a). Carbohydrates with a low glycemic index (GI) result in slow release of glucose into the bloodstream after digestion (Little et al., 2009a). This may be of benefit if consumed before endurance exercise since low-GI carbohydrates would supply a sustained source of energy to contracting muscle (Little et al., 2009b). The low insulin response with consumption of low-GI carbohydrates may also be beneficial for substrate metabolism because high insulin levels inhibit fat lipolysis and oxidation (Horowitz et al., 1997) and, therefore, promote carbohydrate oxidation. Thus, the consumption of low-GI carbohydrates may also promote sparing of muscle glycogen during endurance exercise and improve performance (Little et al., 2010).

Whether lower-GI carbohydrates can actually provide greater benefit for exercise performance compared to higher-GI carbohydrates is an area of controversy. Two recent meta-analyses had contrasting conclusions, with one showing a statistically significant benefit

\footnotetext{
1 - College of Kinesiology, University of Saskatchewan, 87 Campus Dr., Saskatoon SK, Canada, S7N 5B2.

2 - College of Pharmacy and Nutrition, University of Saskatchewan, Saskatoon, Canada, S7N 2Z4.
}

Authors submitted their contribution to the article to the editorial board.

Accepted for printing in the Journal of Human Kinetics vol. 66/2019 in March 2019. 
$(\mathrm{p}=0.001)$ of pre-exercise lower-GI meals/beverages for improving performance over high GI meals/beverages (Heung-Sang Wong et al., 2017) and the second showing no differences in performance between GI conditions (Burdon et al., 2017). Each meta-analysis used similar criteria for their included studies; however, these studies differed slightly between each meta-analysis. The meta-analysis methods differed with one using a more conservative model (i.e. random effects; Burdon et al., 2017) and one using a more liberal model (i.e. fixed effects), which is more likely to result in a statistically significant difference (Heung-Sang Wong et al., 2017). Most importantly the review by Burdon et al. (2017) performed a separate meta-analysis for each type of performance outcome (i.e. time trial, sub-maximal exercise followed by a time trial, and time-toexhaustion); whereas the review by Heung-Sang Wong et al. (2017) standardized all performance outcomes and combined them into one large metaanalysis. The latter approach includes more studies per meta-analysis and therefore has greater statistical power, which could account for their finding of statistically significant performance benefits for lower-GI versus higher-GI carbohydrates.

Lentils have a very low GI and when consumed before endurance exercise they enhance metabolism (i.e. fat oxidation is increased, carbohydrate oxidation is reduced) compared to other carbohydrate sources (Bennett et al., 2012; Little et al., 2009b). This is most likely due to the smaller release of insulin after a low-GI meal because lower insulin levels result in greater fatty acid oxidation (Horowitz et al., 1997). Consuming boiled lentils is not very palatable (Little et al., 2009a, b); therefore, we sought to determine whether lentils incorporated into a sport nutrition bar would offer similar benefits. Our objective was to determine whether a low-GI lentil-based sport nutrition bar consumed prior to exercise could optimise metabolism and exercise performance compared to a sport nutrition bar with a moderate GI.

Moderate to high-GI carbohydrates are generally promoted for recovery from endurance exercise because they enhance glycogen resynthesis after endurance exercise, most likely because they stimulate greater insulin release, which promotes glucose uptake into muscle (Burke et al., 1993). However, when meals of different GIs are compared during recovery after strenuous exercise, studies indicate either superiority of lowGI meals or no difference between meals of different GI on subsequent exercise performance (Brown et al., 2013; Erith et al., 2006; Stevenson et al., 2005). Low-GI meals may promote either greater sparing of glycogen during the recovery exercise session or greater sparing of other important intra-muscular substrates, such as intramuscular triglycerides allowing for superior performance during the recovery exercise session (Stevenson et al., 2009; Trenell et al., 2008). We hypothesized a low-GI lentil-based sports nutrition bar consumed before exercise would enhance fat oxidation, reduce carbohydrate oxidation, and enhance endurance performance compared to a moderate-GI bar and that a low GI bar consumed during recovery from exhaustive exercise would be superior to a moderate-GI bar for improving next-day exercise performance.

\section{Methods}

\section{Participants}

Eight males and three females with a moderate level of cycling experience were recruited $(26 \pm 6 \mathrm{y}, 178 \pm 3 \mathrm{~cm}, 72.9 \pm 4.0 \mathrm{~kg}$, and $\left.\mathrm{VO}_{2 \text { peak }} 51.4 \pm 1.6 \mathrm{~mL} / \mathrm{kg} / \mathrm{min}\right)$. The study followed ethical guidelines consistent with the Declaration of Helsinki; The University of Saskatchewan Research Ethics Board approved the study protocol, and participants provided signed informed consent.

\section{Measures, Design, and Procedures}

On their first visit to the laboratory, participants completed a cycle ergometer (Monark, Ergomedic $874 \mathrm{E}$, Sweden) protocol to determine peak oxygen consumption $\left(\mathrm{VO}_{2 \text { peak }}\right)$. Participants were instructed to pedal at $80 \mathrm{rpm}$. After a 5 -min warm-up, the initial resistance was set at $0.5 \mathrm{kp}$ for females and 1-1.5 $\mathrm{kp}$ for males, then increased every 2 minutes by $0.5 \mathrm{kp}$ until the participant could not keep a pedaling cadence of $70 \mathrm{rpm}$. Throughout testing, $\mathrm{VO}_{2}$ was recorded by open circuit indirect calorimetry (Vmax Series 29 Calorimeter, SensorMedics, USA). The heart rate was measured continuously using a Polar $610 \mathrm{i}$ heart rate monitor (Polar Electro Oy, Kempele, Finland). Criteria that were used for determining $\mathrm{VO}_{2 \text { peak }}$ included achieving a plateau in $\mathrm{VO}_{2}$, respiratory exchange rate more than 1.15 , and 95 
percent of the age-predicted maximum heart rate (Howley et al., 1995). Following the $\mathrm{VO}_{2 \text { peak }}$ test, participants were familiarized with performance testing to be used in the study. This involved a 7 $\mathrm{km}$ time trial where participants cycled as fast as possible at an intensity corresponding to $65 \%$ $\mathrm{VO}_{2 \text { peak. }}$. During this familiarization test and all performance tests subsequently used in the study, participants were blinded to the elapsed time, speed, and cadence by masking the cycle display screen. Participants were allowed to view their elapsed distance during the test. A week later, participants performed a familiarization test on the cycle ergometer that simulated the complete testing protocol. This involved cycling at an intensity corresponding to $65 \% \mathrm{VO}_{2 \text { peak }}$ for 75 minutes, followed by a $7 \mathrm{~km}$ time trial, similar to the first visit. The exercise test was designed to simulate the distance that cyclists would typically cover during an Olympic-distance triathlon (i.e. 40 $\mathrm{km})$.

Using a randomized, counterbalanced, cross-over design, participants consumed $1.5 \mathrm{~g} / \mathrm{kg}$ available carbohydrate from a low-GI lentil bar (the Genki Bar; Genki Bar Foods Inc., Winnepegosis, Manitoba, Canada), a moderate-GI bar (PowerBar Harvest Inc., Berkeley, California) or consumed a placebo (Diet Flavored Jell-O) after an overnight fast, and one hour before endurance cycling for 75 minutes at an intensity that corresponded to $65 \%$ of peak $\mathrm{VO}_{2}$, followed by a 7 $\mathrm{km}$ time trial. The same amount of the bar (or a placebo) was consumed during the first 30 minutes of recovery after exercise, except the PowerBar Recovery was used instead of the PowerBar Harvest. Participants then performed the same 7 $\mathrm{km}$ time trial the next morning, 24 hours after the first time trial, as an assessment of recovery. The PowerBar Harvest was chosen as a comparison bar before exercise because of its similar macronutrient composition as the lentil bar. The PowerBar Recovery was chosen as a comparison bar for recovery because it is one of the leading bars on the market promoted for recovery from endurance exercise. Diet Jell-O was chosen as a placebo because there are no calorie-free sports nutrition bars. Participants were told the diet Jell-O was a sports gel for endurance performance. Macronutrient composition of all the bars is listed in Table 1.

We previously evaluated the GI of all three bars and determined the lentil bar to have a low GI of 47, which was significantly lower than the Harvest PowerBar ${ }^{\mathrm{TM}}(\mathrm{GI}=56)$ and the PowerBar ${ }^{\mathrm{TM}}$ Recovery $(G I=58)$ (Chilibeck et al., 2011). Both Powerbars $^{\mathrm{TM}}$ were classified as having a "moderate" GI. Each feeding condition was separated by at least one week. Females performed each condition one month apart so that they were always in the same phase of their menstrual cycle. The individual administering the exercise tests was blinded to all feeding conditions by having a separate researcher, who was not involved in data collection to prepare the bars or the placebo before the tests and having the participant consume the bars or placebo in a separate room than the researcher performing the exercise tests. The participants were blinded to the bar conditions by removal of the bar wrappers; however, most participants could discern the difference between the bars based on their appearance, especially the PowerBar $^{\mathrm{TM}}$ Recovery because it is coated in chocolate, unlike the other bars. Participants were unaware of the hypotheses of the study.

Respiratory gases were collected from the $10^{\text {th }}$ to the $20^{\text {th }}$ minute and the $60^{\text {th }}$ to the $70^{\text {th }}$ minute during the 75 minutes of constant-load cycling to estimate fat and carbohydrate oxidation. Oxygen consumption $\left(\mathrm{VO}_{2}\right)$, expired carbon dioxide $\left(\mathrm{VCO}_{2}\right)$ and Respiratory Exchange Ratio (RER) were calculated breath-by-breath using opencircuit indirect calorimetry (Vmax Series 29 Calorimeter; Sensor Medics). Carbohydrate and fat oxidation were based on calculations for moderate to high-intensity exercise (Jeukendrup and Wallis, 2005). Rating of perceived exertion (RPE) was measured by using the modified Borg scale (i.e. scale of 1 to 10 ) at the $25^{\text {th }}, 50^{\text {th }}$, and $75^{\text {th }}$ minute of cycling and at the end of the time trial (Borg, 1973).

Capillary blood was drawn from the fingertip and assessed using two different meters (AccuCheck Compact Plus and Accutrend GC; Roche Diagnostics, Sarstedt, Nümbrect, Germany) to measure glucose and lactate levels, respectively, at the $5^{\text {th }}, 15^{\text {th }}, 30^{\text {th }}$, and $60^{\text {th }}$ min after consuming nutrition bars or the placebo, and after 25,50 , and $70 \mathrm{~min}$ cycling and at the end of the $7 \mathrm{~km}$ time trial. Venous blood samples from an antecubital vein were collected at three times (i.e., an hour after consumption of the nutrition bars or placebo, after $75 \mathrm{~min}$ cycling, and following the $7 \mathrm{~km}$ time trial) for assessment of non-esterified fatty acids 
(NEFA), insulin, and catecholamines. The blood samples were maintained in $10 \mathrm{~mL}$ tubes (BD Vacutainer SST, Company, City) for 30 minutes to clot. Serum was separated by centrifugation for 1012 minutes at $3500 \mathrm{rpm}$ and stored at $-80^{\circ} \mathrm{C}$. Insulin, epinephrine, and norepinephrine were determined using an ELISA according to the manufacturer's directions (Insulin EI, Alpco Diagnostics, City; 2cat EIA, Alpco Diagnostics). The serum NEFA assay was performed using a protocol with an oleic acid standard solution as per the manufacturer's directions (NEFAHR (2), Wako Diagnostics). All assays for an individual participant were performed on the same plate to eliminate interassay variability. The intra-assay $\mathrm{CVs}$ for insulin, catecholamines, and NEFA were $<10 \%$.

To minimize any possible influences of diet, physical activity, and sleep variability on study measurements, participants were asked to complete a 24-hour diet, physical activity, and sleep record the day before the first condition and the day of the first condition. The logs were photocopied and returned to participants. Each participant was instructed to repeat the same diet, physical activity, and sleep pattern for the subsequent conditions. Water intake during the exercise tests was matched across conditions. The participant was allowed to drink as much water as they wanted during the first condition and then given the same amount of water during the other conditions.

\section{Statistical Analysis}

All statistical analyses were performed using SPSS for McIntosh (SPSS Version 20; SPSS Inc., Delaware). A two-factor repeated-measures ANOVA (meal and time) was used to assess differences in blood glucose, blood lactate, RER, carbohydrate oxidation, fat oxidation, serum insulin, NEFA, catecholamines (i.e. epinephrine and nor-epinephrine) and RPE. A one factor (meal) repeated measures ANOVA was used to assess time trial performance for the first and second day (i.e. recovery day) of testing. Where significance was noted, an LSD post hoc test was used to determine differences between pairs of means. The level of significance was set at $p<0.05$. Results are presented as means $\pm \mathrm{SD}$.

\section{Results}

Both sports bar conditions elicited a significantly better time trial performance on day 1 than the placebo condition ( $p=0.001$; Figure 1A). The sport bar conditions were not different from each other. The next-day recovery time trial performance was significantly improved in the low-GI condition compared to the moderate-GI bar and the placebo $(p=0.006$; Figure $1 \mathrm{~B})$.

A trend for a condition main effect for RPE was present $(p=0.069$; low-GI bar $=6.3 \pm 0.7$, moderate-GI bar $=6.4 \pm 0.8$; placebo $=6.7 \pm 0.6$ ) . There was no meal $x$ time interaction. As expected, a significant time main effect $(p<0.001)$ was observed with RPE increasing during the exercise (data not shown).

Plasma glucose between low-GI and moderate-GI conditions did not differ throughout the postprandial period and exercise time. As expected, plasma glucose was significantly lower in the placebo condition compared to the low-GI and moderate-GI conditions at all time-points during the postprandial period $(p=0.0001)$ (Figure 2A).

Carbohydrate and fat oxidation rates were similar in the low- and moderate-GI conditions. Carbohydrate oxidation was lower $(p=0.02)$ and fat oxidation higher $(p=0.0001)$ in the placebo condition versus both sport nutrition bars (Table 2).

No significant difference in the respiratory exchange ratio was apparent between the nutrition bar conditions; however, the respiratory exchange ratio was lower in the placebo condition versus sports bar conditions ( $p=0.0001$; Table 2).

There was a condition by time interaction, where the insulin level during the low-GI condition after 75 min cycling was significantly lower than the insulin level in the moderate-GI condition $(p=0.025)$. The insulin response with the placebo was lower than the other conditions at the start of exercise and after the $7 \mathrm{~km}$ time trial, and was lower than the moderate-GI condition after 75 min cycling $(p=0.005)$ (Table 3$)$.

NEFA levels were significantly higher after $75 \mathrm{~min}$ cycling and the $7 \mathrm{~km}$ time trial compared to the onset of cycling across the three conditions (time main effect; $p=0.0001$ ). Also NEFA concentrations were significantly higher in the placebo condition versus the low-GI and moderate-GI conditions across time points (condition main effect; $p=0.002$, Table 3).

The concentrations of epinephrine and norepinephrine were similar in all experimental 
main effect, with catecholamines increasing over the exercise test ( $p=0.0001$, Table 3$)$.

\begin{tabular}{|c|c|c|c|}
\hline \multirow{2}{*}{\multicolumn{4}{|c|}{$\begin{array}{l}\text { Table } 1 \\
\text { Characteristics of the nutrition bars for a } 70 \mathrm{~kg} \text { participant }\end{array}$}} \\
\hline & & & \\
\hline Description & Low GI bar & $\begin{array}{l}\text { Moderate GI } \\
\text { bar }\end{array}$ & $\begin{array}{ll}\begin{array}{l}\text { Moderate } \\
\text { (Recovery) }\end{array} & \text { GI } \\
\end{array}$ \\
\hline Energy (kcal) & 830 & 756 & 910 \\
\hline Fat $(\mathrm{g})$ & 19 & 17 & 35 \\
\hline $\mathrm{CHO}$ total $(\mathrm{g})$ & 127 & 119 & 105 \\
\hline $\mathrm{CHO}$ available (g) & 105 & 105 & 105 \\
\hline Protein $(\mathrm{g})$ & 39 & 35 & 42 \\
\hline Glycemic index & 47 & 56 & 58 \\
\hline
\end{tabular}

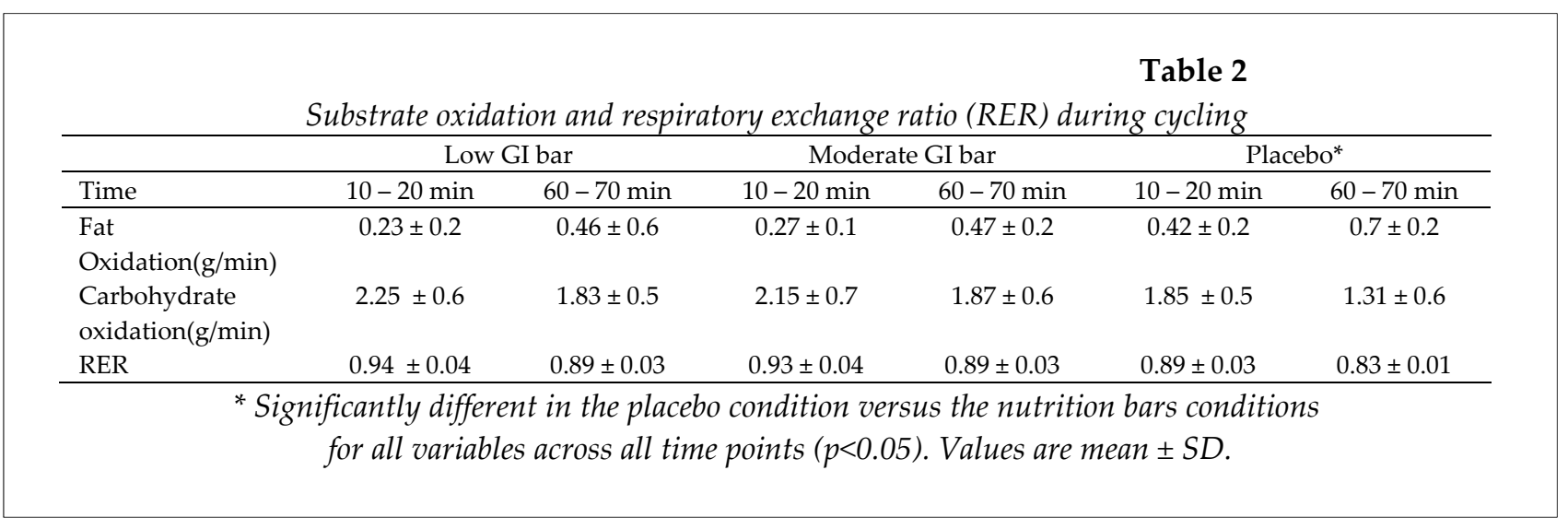

Table 3

Insulin, non-esterified fatty acids (NEFA), epinephrine, and norepinephrine concentrations in the three conditions.

\begin{tabular}{lccccccccc}
\hline Condition & \multicolumn{3}{c}{ Low GI bar } & \multicolumn{3}{c}{ Moderate GI bar } & \multicolumn{3}{c}{ Placebo } \\
\cline { 2 - 9 } & $\begin{array}{c}\text { Onset } \\
\text { of } \\
\text { cycling }\end{array}$ & $75 \mathrm{~min}$ & $\begin{array}{c}\text { After } 7 \\
\mathrm{~km}\end{array}$ & $\begin{array}{c}\text { Onset of } \\
\text { cycling }\end{array}$ & $75 \mathrm{~min}$ & $\begin{array}{c}\text { After } 7 \\
\mathrm{~km}\end{array}$ & $\begin{array}{c}\text { Onset of } \\
\text { cycling }\end{array}$ & $75 \mathrm{~min}$ & After 7 km \\
\hline $\begin{array}{l}\text { Insulin } \\
\text { (mmol/L) }\end{array}$ & $30 \pm 16^{\mathrm{a}}$ & $8 \pm 9 \mathrm{~b}$ & $17 \pm 27 \mathrm{a}$ & $36 \pm 24^{\mathrm{a}}$ & $15 \pm 17$ & $16 \pm 17 \mathrm{a}$ & $6 \pm 5$ & $7 \pm 11^{\mathrm{b}}$ & $3 \pm 2$ \\
NEFAc, & $0.29 \pm$ & $0.67 \pm$ & $0.48 \pm$ & $0.24 \pm$ & $0.7 \pm$ & $0.57 \pm$ & $0.4 \pm 0.2$ & $1 \pm 0.4$ & $0.87 \pm 0.3$ \\
$\begin{array}{l}\text { (mmol/L) } \\
\text { Epinephrine }\end{array}$ & 0.1 & 0.2 & 0.2 & 0.01 & 0.4 & 0.4 & & & \\
(nmol/L) & $0.2 . \pm$ & $0.6 \pm$ & $1.8 \pm$ & $0.18 \pm$ & $0.5 \pm$ & $1.4 \pm$ & $0.2 \pm 0.1$ & $0.8 \pm 0.6$ & $1.3 \pm 0.8$ \\
$\begin{array}{l}\text { Norepinephrine } \\
\text { (nmol/L)e }\end{array}$ & 0.1 & 0.3 & 1.3 & 0.12 & 0.3 & 1.2 & & & \\
\hline
\end{tabular}

Values are mean $\pm S D$ of participants.

a Different compared to the placebo $(p<0.01)$

${ }^{b}$ Different compared to the moderate-GI condition

cTime main effect, 75 min and post-time $7 \mathrm{~km}$ time trial different than onset of cycling $(p<0.01)$

${ }^{d}$ Condition main effect, placebo different than low- and moderate-GI bar across time points $(p<0.01)$

eTime main effect, values for epinephrine and norepinephrine increased across all time points $(p<0.01)$ 
Figure 1

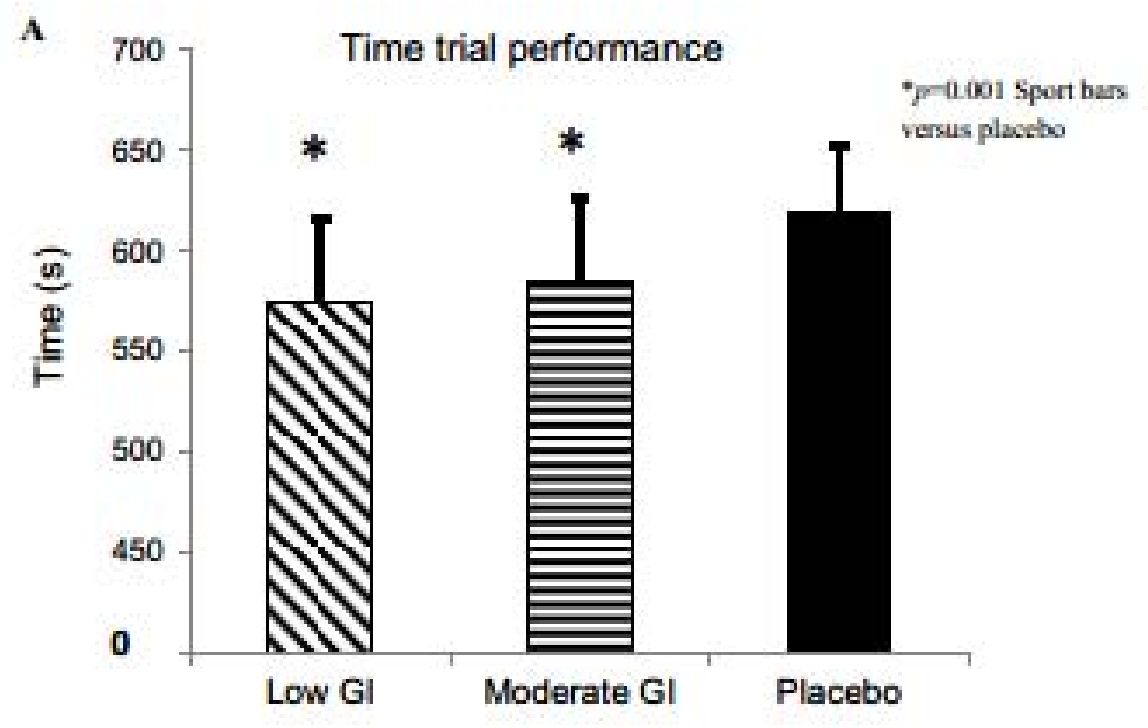

B

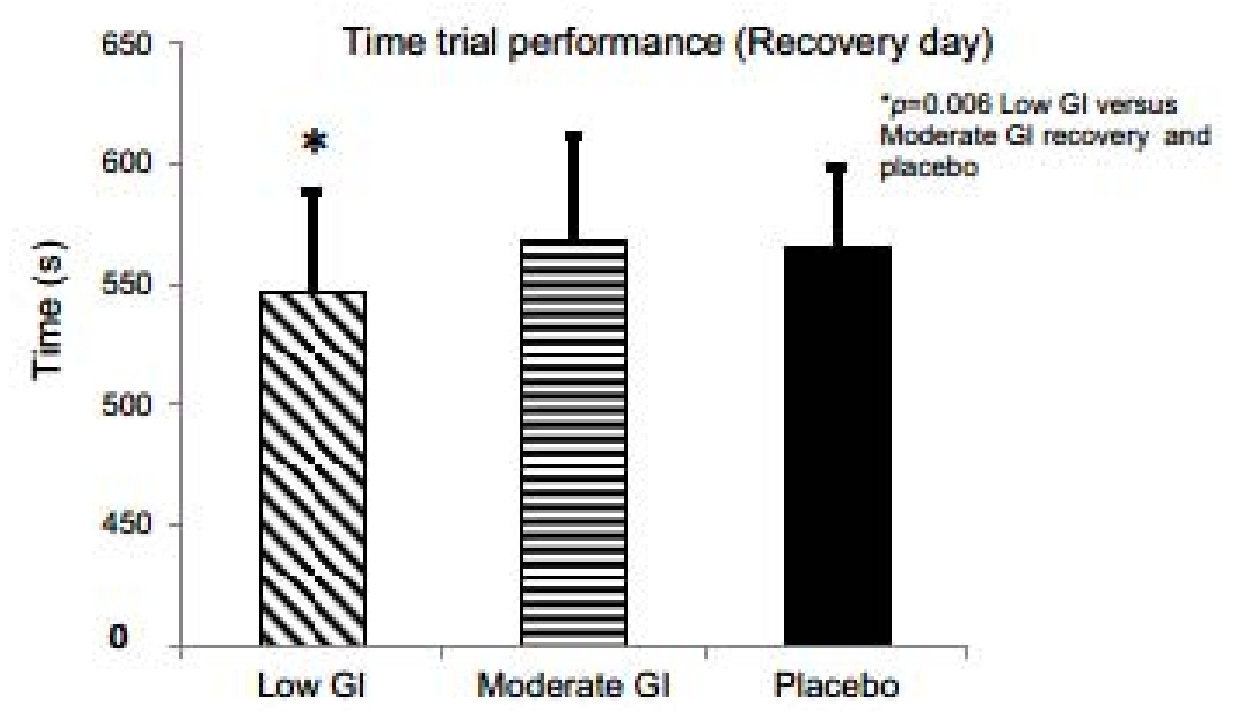

Figure 1

A) Day 1 time trial performance in the three conditions. B) Time trial performance for the day after exercise (recovery day) in the three conditions.

Values are mean and error bars are $S D$. 
Figure 2

$\boldsymbol{A}$

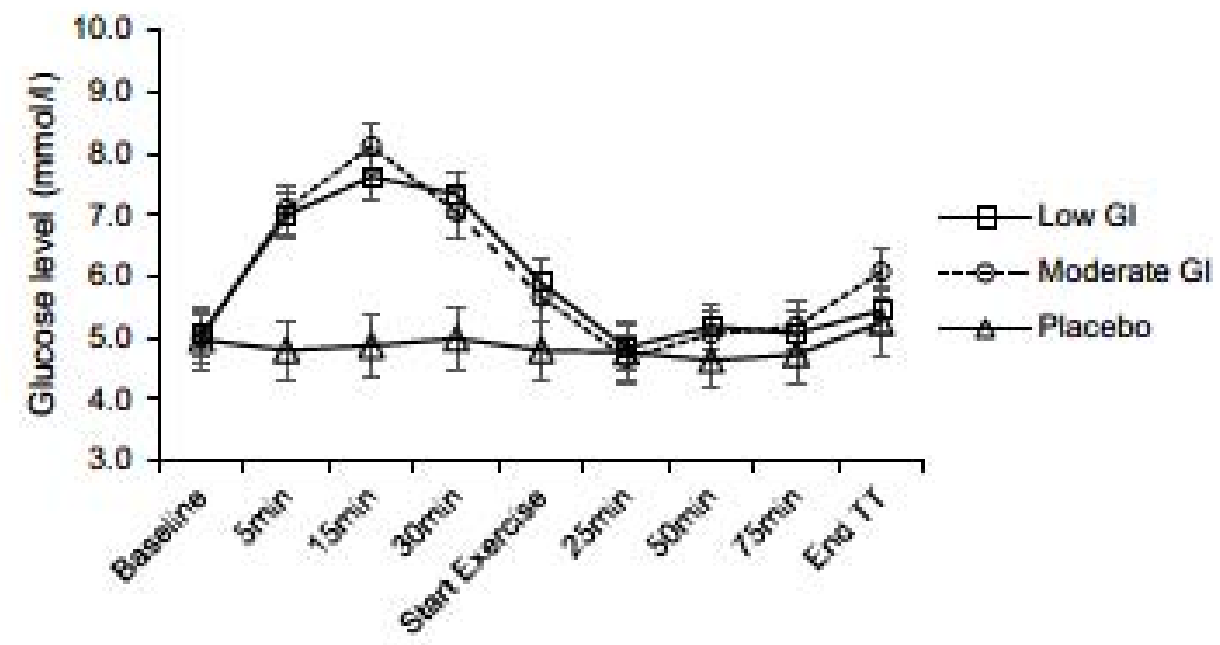

B

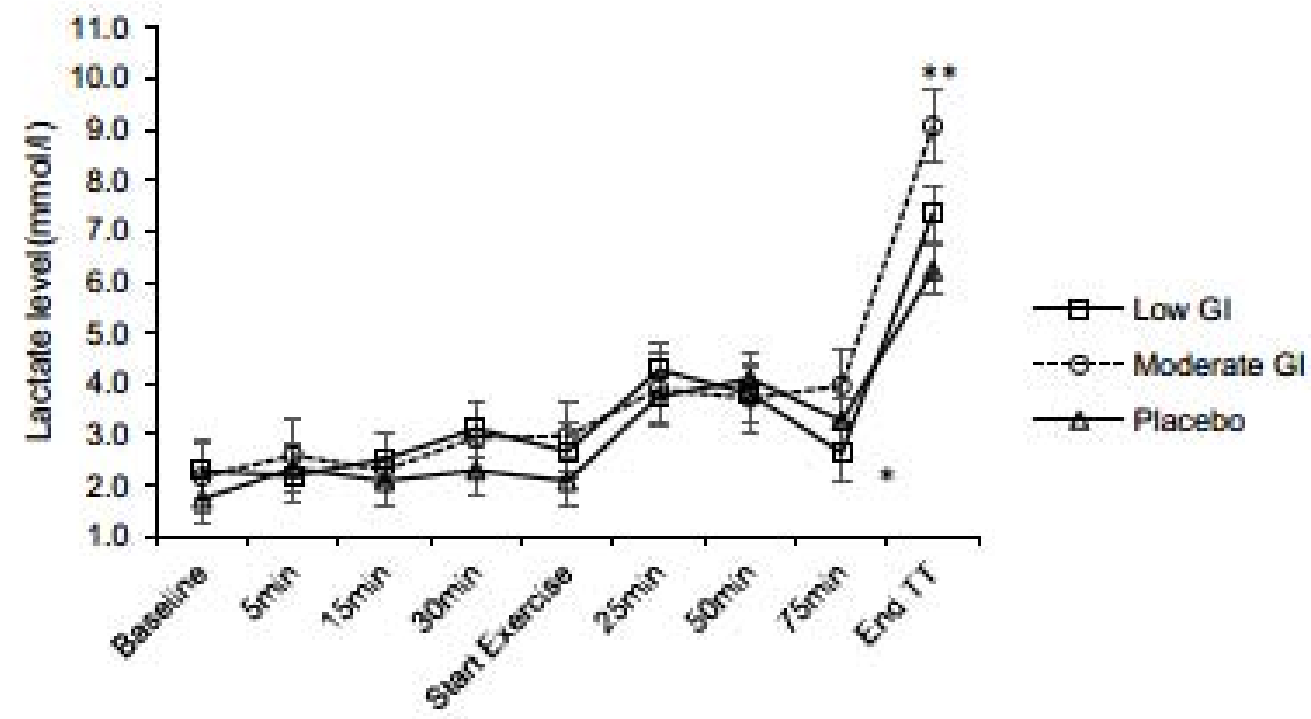

Figure 2

A) Plasma glucose concentration and B) lactate vs. time, at: baseline, postprandial and during exercise.

Values are mean and error bars are SD.

Blood glucose was lower in the placebo compared to the bar conditions $(p<0.01)$

*Blood lactate was higher in the moderate-GI condition versus the low-GI condition $(p<0.01)$

**Blood lactate was higher in the moderate-GI condition versus the low-GI and placebo conditions $(p<0.01)$ 
The data shown in Figure 2B illustrate the impact of the low-GI and moderate-GI bars and the placebo on blood lactate levels during the cycling test and after the $7 \mathrm{~km}$ time trial. A condition by time interaction was present with blood lactate levels significantly higher after $75 \mathrm{~min}$ of exercise in the moderate-GI compared to the low-GI condition $(p=0.003)$. Blood lactate was also higher after the $7 \mathrm{~km}$ time trial in the moderate-GI compared to low-GI and placebo conditions $(p=$ $0.003)$.

All participants complied with instructions to follow similar dietary, sleep, and physical activity patterns across conditions. This was done by checking the initial log completed on the first condition with participants on subsequent conditions. Timing of meals and meal composition before exercise tests and during recovery were similar across conditions with mean daily energy intake of 2465 (SD 860) kcal, protein intake of 109 (SD 46) g, carbohydrate intake of 305 (SD 117) g, and fat intake of 88 (SD 39) g.

\section{Discussion}

The most important finding of this study was that the consumption of bars of differing GI improved performance compared to the placebo when consumed an hour before exercise; however, there was no difference between the bars of a different GI. Consumption of a low-GI bar during recovery was most effective for improving nextday performance. To the best of our knowledge, this study is the first to assess the effect of sport nutrition bars of different GIs on exercise performance, metabolic responses to exercise, and recovery from exercise. This is important given that many sports nutrition bars are commercially available promoting improved exercise performance and recovery.

The consumption of low-GI compared to moderate- or high-GI carbohydrates results in lower and more stable glucose and insulin response. Therefore, low-GI foods could be superior for stimulating fat oxidation and reducing carbohydrate oxidation during exercise because insulin inhibits fat oxidation (Horowitz et al., 1997). The blood glucose response to the moderateGI bar in our study, however, was not significantly different than the low-GI bar (Figure 2a), which differed from our previous determination of a significantly higher glucose response in the
moderate-GI bar (Chilibeck et al., 2011). The differences in findings could be associated with the more precise assessment of the GI in our previous study which evaluated multiple glucose measurements across each time point and was based on the glucose area under the curve over 2 hours. The current study only assessed glucose once at each time point and assessed glucose response for one hour. Although GIs of the nutrition bars were significantly different, the GIs (47 versus 56) may not have been different enough to elicit significantly different metabolic responses. The insulin response to the two bars was similar, with the moderate-GI bar eliciting a higher insulin response only at the 75th minute mark of exercise.

A lack of difference in fat and carbohydrate oxidation, mobilization of fatty acids, and catecholamine response between the bar conditions was different from other studies comparing low-GI lentils to high-GI meals (Bennett et al., 2012; Little et al., 2009b; Moore et al., 2010). Again this may be due to the relatively small difference in GIs between the bars. The differences in the GI between the bars in the current study was only 9 GI units; whereas the difference in the GI of other studies detecting differences in substrate oxidation had differences ranging from 36 to 91 GI units for lower versus higher-GI conditions (Bennett et al., 2012; Little et al., 2009b). Males and females respond differently regarding fat oxidation during endurance exercise (Tarnopolsky, 2008) and therefore the inclusion of females may have distorted our data. A limitation of our study is the inclusion of only a small number of females, which did not allow us to include sex as a factor in our analysis; however, when we excluded females from our analysis, there were still no differences between GI conditions for substrate oxidation.

Lactate production late in the exercise session was lower during the low-GI condition (Figure 2b), which may have indicated a smaller use of glycogen during anaerobic metabolism. This is consistent with other studies showing a higher blood lactate concentration during exercise when high- compared to low-GI foods are consumed before exercise (Karamanolis et al., 2011; Wee et al., 1999, 2005). Wee et al. (2005) suggested that a greater accumulation of muscle and blood lactate during treadmill running was accompanied by reductions of muscle glycogen during a high-GI 
trial. In contrast with this, other studies failed to find any significant difference in lactate accumulation when food with different GIs was consumed prior to continuous exercise (Kirwan et al., 1998; Thomas et al., 1991).

A lower use of glycogen through anaerobic metabolism may have contributed to the superior performance during the low-GI bar condition versus the moderate-GI condition during the nextday performance. In agreement with this, Stevenson et al. (2009) reported that a low-GI diet consumed in the $24 \mathrm{~h}$ period following continuous heavy exercise led to a greater endurance capacity during steady state exercise in the post-absorptive state the following day compared with a high-GI carbohydrate diet. Moreover, Trenell et al. (2008) showed that using a low-GI recovery diet may spare intramuscular triglyceride utilised during recovery exercise performance, whereas a high-GI diet reduced NEFA availability throughout exercise and increased reliance on intramuscular lipids as an energy source during moderate intensity exercise. Intramuscular triglyceride, in addition to glycogen, may be an important energy source for skeletal muscle during endurance exercise.

One of the unique aspects of this study was testing of different sport nutrition bars for the subsequent exercise metabolism and performance. Consuming nutrition bars has become popular among athletes since they are convenient and well portion-controlled (Rauch et al., 1999). In the present study we provided multiple nutrition bars instead of a single bar in order to meet recommended carbohydrate intake prior to endurance exercise. To the best of our knowledge, this study was the first to investigate the effect of consuming multiple nutrition bars on exercise performance; whereas, most previous nutritional bar studies failed to show beneficial results because the amount of bars consumed was too few based on previous research of how much carbohydrate is needed to improve performance (Kolkhorst et al., 1998; Oliver and Tremblay, 2002; Rauch et al., 1999). A limitation of the study was that changes in the muscular glycogen and intramuscular triglyceride stores were not measured following the first time trial and the subsequent time trial the next day to disclose the possible metabolic reasons for better performance in the low-GI condition.

In summary, low- and moderate-GI bars elicited similar metabolic responses and had similar benefits for endurance performance; however, a low-GI bar enhanced recovery as indicated by superior next-day exercise performance. This might be attributable to lower anaerobic glycogenolysis and, therefore, sparing of muscle glycogen late in the initial exercise session. Irrespective of the mostly similar responses in the current study to low- and moderate-GI bars, consumption of low-GI bars by athletes may have long-term nutritional health benefits compared to those bars that elicit an undesirable glucose response (Tegelman et al., 1996).

\section{Practical Recommendations}

Low or moderate-GI sport nutrition bars, when consumed in adequate amounts to meet guidelines for pre-exercise carbohydrate consumption (i.e. $\geq 1.0 \mathrm{~g} / \mathrm{kg}$ available carbohydrate) are beneficial for improving endurance exercise performance. When consumed during recovery, low-GI sport nutrition bars appear to provide the greatest benefit with regard to the next-day endurance exercise performance.

\section{Acknowledgements}

This study was funded by Agriculture and Agri-food Canada and the Saskatchewan Pulse Growers..

\section{References}

Bennett CB, Chilibeck PD, Barss T, Vatanparast H, Vandenberg A, Zello GA. Metabolism and performance during extended high-intensity intermittent exercise after consumption of low- and high-glycaemic index pre-exercise meals. Br J Nutr, 2012; 108: 81-90

Borg GA. Perceived exertion: a note on history and methods. Med Sci Sports Exer, 1973; 5: 90-93

Brown L, Midgley AW, Vince RV, Madden LA, McNaughton LR. High versus low glycemic index 3-h 
recovery diets following glycogen-depleting exercise has no effect on subsequent 5-km cycling time trial performance. J Sci Med Sport, 2013; 16: 450-454

Burdon CA, Spronk I, Lun Cheng H, O'Connor HT. Effect of glycemic index of a pre-exercise meal on endurance exercise performance: a systematic review and meta-analysis. Sports Med, 2017; 47: 1087-1101

Burke LM, Collier GR, Hargreaves M. Muscle glycogen storage after prolonged exercise: Effect of the glycemic index of carbohydrate feedings. J Appl Physiol, 1993; 75: 1019-1923

Chilibeck PD, Rooke J, Zello GA. Development of a lentil-based sports nutrition bar. Appl Physiol Nutr Metab, 2011; 36: 308-309

Erith S, Williams C, Stevenson E, Chamberlain S, Crews P, Rushbury I. The effect of high carbohydrate meals with different glycemic indices on recovery of performance during prolonged intermittent highintensity shuttle running. Int J Sport Nutr Exerc Metab, 2006; 16: 393-404

Heung-Sang Wong S, Sun F-H, Chen Y-J, Li C, Zhang Y-J, Ya-Jun Huang W. Effect of pre-exercise carbohydrate diets with high vs low glycemic index on exercise performance: a meta-analysis. Nutr Rev, 2017; 75: 327338

Horowitz JF, Mora-Rodriguez R, Byerley LO, Coyle EF. Lipolytic suppression following carbohydrate ingestion limits fat oxidation during exercise. Am J Physiol, 1997; 273: 768-775

Howley ET, Basset DR, Welch HG. Criteria for maximal oxygen uptake: review and commentary. Med Sci Sports Exerc, 1995; 27: 1292-1301

Jeukendrup AE, Wallis GA. Measurement of substrate oxidation during exercise by means of gas exchange measurements. Int J Sport Med, 2005; 26(1): S28-37

Karamanolis IA, Laparidis KS, Volaklis KA, Douda HT, Tokmakidis SP. The effects of pre-exercise glycemic index food on running capacity. Int J Sports Med, 2011; 32: 666-671

Kirwan JP, O'Gorman D, Evans WJ. A moderate glycemic index meal before endurance exercise can enhance performance. J Appl Physiol, 1998; 84: 53-59

Kolkhorst FW, MacTaggart JN, Hansen MR. Effect of a sports food bar on fat utilisation and exercise duration. Can J Appl Physiol, 1998; 23: 271-278

Little JP, Chilibeck PD, Bennett C, Zello GA. Food for endurance: The evidence, with a focus on the glycaemic index. CAB Reviews: Perspectives in Agriculture, Veterinary Science, Nutrition and Natural Resources, 2009a; 4(058): 1-13

Little JP, Chilibeck, PD, Ciona D, Forbes S, Rees H, Vandenberg A, Zello GA. Effect of low- and high-glycemicindex meals on metabolism and performance during high-intensity, intermittent exercise. Int J Sport Nutr Exerc Metab, 2010; 20: 447-456

Little JP, Chilibeck PD, Ciona D, Vandenberg A, Zello GA. The effects of high and low glycemic index foods on high intensity intermittent exercise. Int J Sports Physiol Perform, 2009b; 4: 367-380

Moore LJ, Midgley AW, Thurlow S, Thomas G, Mc Naughton LR. Effect of the glycaemic index of a preexercise meal on metabolism and cycling time trial performance. J Sci Med Sport, 2010; 13: 182-188

Oliver SK, Tremblay MS. Effects of a sports nutrition bar on endurance running performance. J Strength Cond Res, 2002; 16: 152-156

Rauch HG, Hawley JA, Woodey M, Noakes TD, Dennis SC. Effects of ingesting a sports bar versus glucose polymer on substrate utilisation and ultra-endurance performance. Int J Sports Med, 1999; 20: 252-257

Stevenson EJ, Astbury NM, Simpson EJ, Taylor MA, Macdonald IA. Fat oxidation during exercise and satiety during recovery are increased following a low-glycemic index breakfast in sedentary women. $J$ Nutr, 2009; 139: 890-897

Stevenson E, Williams C, McComb G, Oram C. Improved Recovery from Prolonged Exercise Following the Consumption of Low Glycemic Index Carbohydrate Meals. Int J Sport Nutr Exerc Metab, 2005; 15: 333349 
Tarnopolsky MA. Sex differences in exercise metabolism and the role of 17-beta estradiol. Med Sci Sports Exerc, 2008; 40: 648-654

Tegelman R, Aberg T, Eklöf R, Pousette A, Carlström K, Berglund L. Influence of a diet regimen on glucose homeostasis and serum lipid levels of male elite athletes. Metabolism, 1996; 45: 435-441

Thomas DE, Brotherhood JP, Brand JC. Carbohydrate feeding before exercise: Effect of glycemic index. Int J Sports Med, 1991; 12: 180-186

Trenell IM, Stevenson E, Stockmann K, Brand-Miller J. Effect of high and low glycaemic index recovery diets on intramuscular lipid oxidation during aerobic exercise. Br J Nutr, 2008; 99: 326-332

Wee SL, Williams C, Gray S, Horabin J. Influence of high and low glycemic index pre-exercise meals on endurance running capacity. Med Sci Sports Exerc, 1999; 31: 393-399

Wee SL, Williams C, Tsintzas K, Boobis L. Ingestion of a high-glycemic index meal increases muscle glycogen storage at rest but augments its utilization during subsequent exercise. J Appl Physiol, 2005; 99: 707-714

\section{Corresponding author:}

\section{Philip Chilibeck, Ph.D.}

College of Kinesiology

University of Saskatchewan

87 Campus Dr.

Saskatoon SK

Canada, S7N 5B2

Phone: 1-306-966-1072

Fax: 1-306-966-6464

Email: Phil.chilibeck@usask.ca 